\title{
Mit dem Trackball zur präzisen Restauration
}

Welcher Patient wäre davon nicht begeistert: von komfortablen Abformungen ohne schlechten Geschmack und ohne zu unterdrückenden Würgereiz und anschließenden passgenauen, zahnfarbenen und dauerhaften Restaurationen in derselben Sitzung? Das aktuelle chairside CAD/CAM-System CEREC (Sirona, Bensheim), das seit 30 Jahren kontinuierlich weiterentwickelt wird, ermöglicht Zahnärzten, ihren Patienten genau das anzubieten, nämlich eine präzise Restauration in 1 Sitzung einzusetzen.

Anlässlich eines praktischen Kurses am 3. Dezember 2015 in der Sirona Dental Akademie in Bensheim, zu dem einige wenige Fachpressevertreter eingeladen waren, konnte ich, angeleitet durch Produkttrainerin Svenja Hölzel, selbst erfahren, wie praxisnah das Verfahren ist. Unsere Aufgabe war es, den an einem Modell bereits präparierten Zahn 47 mit einer CERECKeramikkrone zu versorgen.

\section{Digitale Abformung}

Nach einigen administrativen Angaben per Mausklick und Trackball (Rollkugel; vergleichbar mit einer kleinen PC-Maus) an der CEREC AC-Aufnahmeeinheit beginnt der prothetische Arbeitsablauf mit der digitalen Abformung mithilfe der CEREC-Omnicam. Ich führe die Kamera, die wie ein Stift zu halten ist, über Zähne und Gingiva. Der Kamerakopf ist klein, sodass ein Scannen im Patientenmund auch im hinteren Seitenzahnbereich realistisch ist. Für Zahn 47 reicht es aus, den präparierten Zahn, einige Nachbarzähne, den Gegenkiefer sowie den bukkalen Biss zu scannen. „Je mehr Sie scannen, desto länger rechnet später das Programm, um die Informationen zu verarbeiten", gibt die Trainerin zu bedenken. Ein Ganzkieferscan empfehle sich z.B., wenn mehrere Restaurationen in einem Kiefer hergestellt werden sollen oder im Bereich der Implantologie für eine Bohrschablone.

Die Kamera vermisst puder- und berührungsfrei direkt die Oberflächen. Dabei lerne ich, dass ein Abstand zwischen Kamerakopf und aufzunehmendem Bereich von etwa $5 \mathrm{~mm}$ sinnvoll ist und sich ausgehend von der Okklusalfläche leichte Kippbewegungen mit der Kamera als Scan-Strategie empfehlen. Während der Aufnahme soll der Zahnarzt-Blick auf den Monitor gerichtet sein. Denn die digitale Abformung ist ein fortlaufender Videoprozess, und am Monitor entsteht während des Scans zeitgleich ein 3-dimensionales virtuelles Modell in Farbe.

Scanne ich nachlässig und erfasse einen Bereich ungenügend, ändert sich das akustisches Signal, der 3D-Aufbauprozess wird unterbrochen. Kein Problem - das System ermöglicht mir, einfach nochmals anzusetzen und „nachzuscannen“. Das Programm fügt die Bereiche nahtlos zusammen. „Es ist eigentlich kinderleicht“, fasst Hölzel auch meinen Eindruck zusammen. Nach rund 10 min sind Präparations-, Gegenkiefer- und bukkale Aufnahme zur Bissregistrierung gemacht.

\section{Konstruieren und Formschleifen} Dann zeichne ich den Präparationsrand ein und definiere die Einschubachse. Optional führt das System eine Präparationsanalyse u.a. hinsichtlich Unterschnitte und Abstand zum Antagonisten durch.

„Biogenerik“ war gestern, nun sorgt „Biokiefer" für passgenaue Restaurationen: In der aktuellen Software CEREC SW 4.4 mit neuen Algorithmen wird der eingescannte Bereich analysiert und als Berechnungsgrundlage verwendet. Das Ergebnis ist ein patientenindividueller Erstvorschlag, der faszinierend perfekt erscheint. Wer selbst noch einmal Hand anlegen will, um die Restauration zu gestalten, hat dafür genügend virtuelles Werkzeug. Das nutze ich, um die Approximalkontakte zu verstärken. Mit der Trainerin an der Seite kann nichts schief gehen. Doch wie steht es um die Lernkurve? Erfahrungsgemäß starten die meisten Zahnärzte mit einer Einzelkrone und planen dafür etwas mehr als 90 min ein. Wichtig ist die Übung. Grundsätzlich erhält ,jeder neue Anwender eine ausführliche Einweisung“, erklärt Hölzel. „Die Hilfefunktion innerhalb der Software unterstützt darüber hinaus während der Gestaltung. Außerdem sind regelmäßige Kurse in der Akademie oder Trainings, z. B. von der Deutschen Gesellschaft für Computergestützte Zahnheilkunde (DGCZ), für das gesamte Team zu empfehlen.“

Schließlich erteile ich mit einem Klick den Auftrag fürs Formschleifen der Krone, für das mir die Schleifmaschine zur Verfügung steht. In diese wird der zu Beginn ausgewählte Keramikblock eingesetzt. Die Maschine positioniert die Instrumente, die

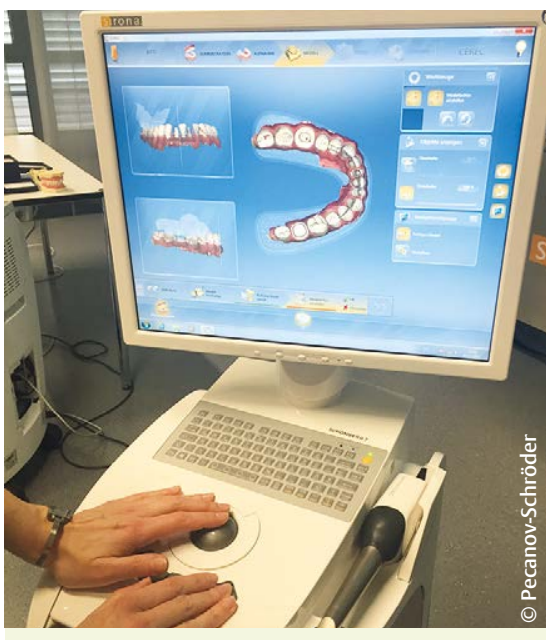

Scannen und Konstruieren mit der mobilen Cartvariante CEREC AC mit Omnicam.

den Block abtasten und „überprüfen“, ob das Material mit meiner Eingabe übereinstimmt. Nach $11 \mathrm{~min}$ ist der Schleifprozess beendet. Ich setze die Restauration auf den Modellzahn und bin von der Passung überzeugt. In der Praxis würde nun das Finalisieren der Restauration erfolgen (Trennstelle reduzieren, Anpassung im Mund, Hochglanzpolitur). Laut einem klinischen Leitfaden („CEREC Basiswissen“ von Dr. Andreas Ender und Prof. Dr. Dr. Albert Mehl) sollen die Approximalflächen vor dem adhäsiven Einsetzen, während $\mathrm{Au}$ ßen- und Okklusalflächen nach dem adhäsiven Einsetzen poliert werden.

\section{Fazit für die Praxis}

CEREC überzeugt und macht durch seine Anwenderfreundlichkeit förmlich Lust auf digital gefertigte Kronen, Inlays, Onlays und mehr in der täglichen Zahnarztpraxis. Wie schnell sich die Investition in das CEREC System (rd. $90000 €$ für die Premiumversion) rechnet, hängt von vielen praxisspezifischen Parametern ab. Doch Rechenbeispiele lassen den Schluss zu, dass bei richtiger Integration in der Praxis das chairside CAD/CAM-System durchaus eine „Win-win-Situation“ sein kann: Das Praxisteam ist zufrieden, wenn die Qualität stimmt und Zeit und Kosten eingespart werden können. Der Patient ist glücklich, wenn er möglichst wenige Termine in Anspruch nehmen muss, die Abläufe für ihn bequem sind und am Ende alles passt. Prima, wenn er seine guten Erfahrungen weiterträgt und neue Patienten in die Praxis kommen - dann funktioniert CEREC sogar als Marketing-Instrument.

Dr. med. dent. Aneta Pecanov-Schröder, Bonn 